\title{
REMARKS ON RECONSTRUCTION OF DEFECTS' DISTRIBUTION BY POSITRON LIFETIME MEASUREMENTS
}

\author{
J. DRYZEK \\ Institute of Nuclear Physics, Radzikowskiego 152, 31-342 Kraków, Poland
}

It is well known that the positron trapping rate obtained from positron lifetime measurements depends upon defect concentrations and trapping efficiency. If a distribution of open volume defects exists, a distribution of trapping efficiency also could be present. As an example, the calculation of trapping efficiency as a function of radius of defects in $\mathrm{Al}$ is presented. The correct explanation of defect size distribution obtained by positron lifetime measurements needs to introduce some additional assumptions about trapping efficiency.

PACS numbers: $78.70 . \mathrm{Bj}$

The traditional deconvolution procedures of experimental positron lifetime spectra allow to obtain only discrete and limited number of positron lifetime components [1]. In such a case the experimental lifetime spectrum is an expression of a sum of a finite number $(n)$ of exponentials convoluted with the instrument resolution function

$$
N_{\exp }(t)=R(t) N_{t} \sum_{i=1}^{n} \lambda_{i} I_{i} \exp \left(-\lambda_{i} t\right)+B,
$$

where $N_{\exp }(t)$ is the experimental raw data, $N_{t}$ is the total count value, $B$ is a background, $\lambda_{i}$ is the inverse of the $i$-th lifetime component $\left(\lambda_{i}=1 / \tau_{i}\right)$ and $I_{i}$ is its intensity or the fraction of positrons annihilating with lifetime $\tau_{i}$.

Usually number of positron lifetime components do not exceed twenty $(n<20)$. In laboratory the deconvolution of the experimental positron lifetime spectrum is performed with two, three or four lifetime components which can give sufficient information about the behavior of positrons in the sample. However, new deconvolution procedures based on the Laplace inversion technique [2] and the quantified maximum entropy method [3] allow to obtain a distribution of positron lifetime from the continuous decay Eq. (1):

$$
N_{\exp }(t)=R(t) N_{t} \int_{0}^{\infty} \mathrm{d} \lambda \lambda I(\lambda) \exp (-\lambda t)+B .
$$


It seems that these new deconvolution procedures can give more information about the history of positrons in solid but they have several limitations e.g. the source correction must be taken precisely because of the unstability of results. Using these procedures nevertheless many authors hope to get the distribution of open volume defects, e.g. clusters of vacancies or pores [4].

It would be very interesting to get such information but it should be remembered that in positron experiments only the positron trapping rate is measured. The concentration of positron traps, e.g. vacancies or their clusters, is related to the trapping rate as follows:

$$
K_{\mathrm{D}}(\tau)=\mu_{\mathrm{D}} C_{\mathrm{D}}(\tau)
$$

where $\mu_{\mathrm{D}}$ is the trapping efficiency.

From the trapping model it is easy to get the relation between intensity $I$ and trapping rate as a function of positron lifetime

$$
K_{\mathrm{D}}(\tau)=I(\tau)\left(\frac{1}{\tau_{1}}-\frac{1}{\tau}\right)
$$

where $\tau_{1}$ is the shortest positron lifetime in positron lifetime spectra. The correction factor in round parentheses is small but it is needed for proper presentation of any experimental data. The relation (3) shows that the dependence of the trapping rate upon positron lifetime may be caused by a size distribution of vacancy concentration and/or the trapping efficiency can also be a function of the defect radius.

One can deduce that trapping efficiency is much more sensitive to the nature and geometry of the vacancy capturing positrons than positron lifetime. But from the experiment it is much more difficult to obtain trapping efficiency than positron lifetime. Therefore, positron lifetime is used for characterization of defects. In Fig. 1 there are presented results of calculations of trapping efficiency as a function of vacancy radius for three temperatures $(T=4 \mathrm{~K}, 300 \mathrm{~K}$ and $1000 \mathrm{~K})$. The calculations were performed using the approach with complex potential described in [5]. In that case it was assumed that $V=-9.9(1+0.047 \sqrt{-1}) \mathrm{eV}$ and other parameters as in Al. From Fig. 1 one can see that trapping efficiency is a complicated function of temperature and defect radius. If some distribution of size of vacancies is reflected by positron lifetime and exists in the samples, it is difficult to assume that trapping efficiency is constant for all vacancies. According to the calculations the change of the trapping efficiency is within one order of magnitude. It is small in comparison with the change of total vacancy concentration but it may be relevant to deduce their distribution.

In these calculations it was assumed that the trapping of positrons is limited only by the transition from a free to a bound state. The trapping efficiency exhibits almost linear dependence on radius of voids when trapping is limited by the diffusion process of positrons to defects. That relation was experimentally confirmed. However, there is experimental evidence that in case of voids (in $\mathrm{Al}$ ) at low temperatures $(77 \mathrm{~K})$ the mechanism controlling positron trapping is the transition whereas at higher temperatures $(300 \mathrm{~K})$ it is the diffusion ([6], [7] and [8]).

Concluding, from positron lifetime spectrum one can deduce only the distribution of the positron trapping rate at defects. Knowing the trapping efficiency, 

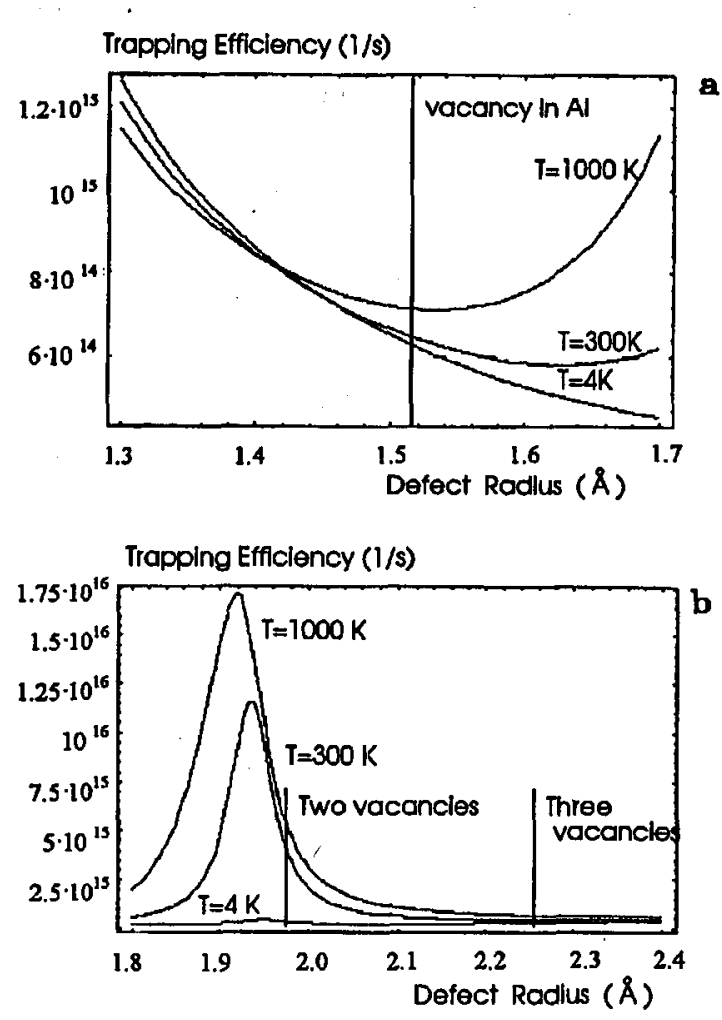

Fig. 1. The dependence of trapping efficiency as a function of vacancy radius (a) and vacancy clusters (b) in $\mathrm{Al}$ for three temperatures: $4 \mathrm{~K}, 300 \mathrm{~K}$ and $1000 \mathrm{~K}$. The vacancy and clusters are calculated using the complex potential $V=-9.9(1+0.047 \sqrt{-1}) \mathrm{eV}$.

and the trapping rate one can obtain the defect concentration. Theoretical calculation and experiments show the complexity of trapping efficiency as a function of geometry, size of defects and external conditions e.g. temperature. The above presented results show that without any additional assumptions, it is difficult to obtain the size distribution of defects from new deconvolution procedures of positron lifetime spectra.

The author would like to thank the State Committee for Scientific Research (Republic of Poland) for supporting this work under research grant No. 2P302 02804.

\section{References}

[1] M. Eldrup, I.K. Mackenzie, B.T.A. McKee, D. Segers, in: Positron Annihilation, Proc. ICPA-8, Eds. L. Dorikens-Vanpraet, M. Dorikens, D. Segers, World Scientific, Singapore 1988, p. 224.

[2] R.B. Gregory, Nucl. Instrum. Methods Phys. Res. A 302, 496 (1991). 
[3] A. Shukla, M. Peter, L. Hoffmann, Nucl. Instrum. Methods Phys. Res. A 335, 310 (1993).

[4] Y.C. Jean, F. Zandiehnadem, Q. Deng, Mater. Sci. Forum 105-110, 1897 (1992).

[5] J. Dryzek, Phys. Status Solidi B 179, 15 (1993).

[6] M. Eldrup, K.O. Jensen, Phys. Status Solidi A 102, 145 (1987).

[7] A. Seeger, Appl. Phys. 4, 183 (1974).

[8] H. Murakami, T. Endo, I. Matsuda, Mater. Sci. Forum 105-110, 1159 (1992). 